\title{
East Asian Primary Care Research Network (EAPCRN)
}

\author{
Created by: RHarry H.x. Wang
}

Version received: 29 March 2020

check for

updates

\begin{abstract}
The East Asian Primary Care Research Network (EAPCRN), initially proposed by Professor Jae-Ho Lee who is the Chairman of the Korean Primary Care Research Group, was officially established during the $22^{\text {nd }}$ World Organization of Family Doctors (WONCA) World Conference - "Primary Care in the Future: Professional Excellence" during 17-21 October 2018 in Seoul, South Korea. The EAPCRN inauguration seminar at WONCA Seoul 2018 was convened by Jae-Ho Lee (Korean Primary Care Research Group; The Catholic University of Korea) and co-convened by Harry H.X. Wang (Sun Yat-Sen University), Takuya Aoki (Kyoto University), and Chyi-Feng Jan (National Taiwan University).
\end{abstract}

\section{HISTORY:}

The East Asian Primary Care Research Network (EAPCRN), initially proposed by Professor Jae-Ho Lee who is the Chairman of the Korean Primary Care Research Group, was officially established during the $22^{\text {nd }}$ World Organization of Family Doctors (WONCA) World Conference - "Primary Care in the Future: Professional Excellence" during 17-21 October 2018 in Seoul, South Korea. The EAPCRN inauguration seminar at WONCA Seoul 2018 was convened by Jae-Ho Lee (Korean Primary Care Research Group; The Catholic University of Korea) and co-convened by Harry H.X. Wang (Sun Yat-Sen University), Takuya Aoki (Kyoto University), and Chyi-Feng Jan (National Taiwan University).

\section{RATIONALE \& RESEARCH HYPOTHESIS:}

It is the vision of the EAPCRN that primary care is a key component of a health care system and many countries have been making efforts through primary care reforms to strengthen the care delivery. Policy decisions often depend more on optimistic theory than on research evidence. Primary care research is a necessary prerequisite if we are dedicated to enhance the role of primary care in health care systems, facilitate the optimal functioning of those health care systems, and to improve the health of populations in the long term. At an international level, the exchange of scientific knowledge and methodologies between researchers with insights of different countries can also provide networking benefits. One highlight of research interests of the EAPCRN is the primary care profiles in each of the different health care systems. The focus is placed on consensus definition, providers, facilities, and payment methods in primary care, as well as the role of primary care in health care system with long-term effects.

\section{FACEBOOK:}

https://www.facebook.com/The-East-Asian-Primary-Care-Research-Network-2103974623167831/

\section{MEMBERS:}

Jae-Ho Lee, MD, MPH, PhD

Professor, College of Medicine, The Catholic University of Korea; Chairman of the Korean Primary Care Research Group (KPCRG)

Takuya Aoki, MD, PhD, MMA

Department of Healthcare Epidemiology/Department of Community Medicine, Kyoto University Graduate School of Medicine, Japan

Shin Yoshida, MD

Chair for Young Doctor Movement in Japan Primary Care Association and the Rajakumar Movement in WONCA APR; Vice Director of lizuka-Kaita Family Medicine Program, Fukuoka, Japan

Chyi-Feng Jan, MD, PhD

Director, Division of Community Medicine, Department of Family Medicine, Medical College, National Taiwan University; Deputy Director, Referral Management Center, National Taiwan University Hospital

Harry H.X. Wang, PhD 
Associate Professor, School of Public Health, Sun Yat-Sen University, Guangzhou, PR China; Honorary Senior Lecturer, General Practice and Primary Care, Institute of Health and Wellbeing, University of Glasgow, UK

\section{EAPCRN SEMINAR CHAIRS:}

Jungkwon Lee, MD, PhD

Overall Chair of Organizing Committee, $19^{\text {th }}$ WONCA Asia Pacific Regional Conference; Family Medicine at Sungkyunkwan University School of Medicine, Korea

Machiko Inoue, MD, MPH, PhD

Professor, Department of Family and Community Medicine, Hamamatsu University School of Medicine, Japan; Director of Shizuoka Family Medicine Training Program, Japan

\section{KEY TIMELINES:}

2018 - Seminar titled "Primary Care Reform and Health Services Research in East Asia - a Seminar for Establishing the East Asian Primary Care Research Network", Seminar No. WK0031 at the WONCA World Conference (Seoul, South Korea, October 2018)

\section{Chair:}

Jungkwon Lee, Family Medicine at Sungkyunkwan University School of Medicine, Korea

\section{Convenor:}

Jae-Ho Lee, The Catholic University of Korea, Seoul, Korea

Co-Convenors:

Harry H.X. Wang, Sun Yat-Sen University, Guangzhou, PRC

Takuya Aoki, Kyoto University, Kyoto, Japan

Chyi-Feng Jan, National Taiwan University, Taipei, ROC

Main task:

To present, compare, and discuss the structure and function of primary care across different health care systems in East Asia, and the need for building the EAPCRN.

Implications:

The seminar will bring together prominent scholars to share a unique combination of health services research updates in evaluating process and outcome of primary care, with the use of well-developed instruments including Primary Care Assessment Tool (PCAT). We expect this seminar can be a stepping stone to establish an East Asian Primary Care Research Network, inspiring cutting-edge research with mutual interest on many aspects.

2019 - Symposium titled "Building primary care in a changing East Asia - a Project on Primary Care System Profiles from the East Asian Primary Care Research Network", Symposium No. SY2-05 at the WONCA Asia Pacific Regional Conference (Kyoto, Japan, May 2019)

Chair:

Machiko Inoue, Hamamatsu University School of Medicine, Japan

Organizer:

Takuya Aoki, Kyoto University, Japan

Symposium No. SY2-0501 Current status and profiles of primary care in South Korea Jae-Ho Lee, Nak-Jin Sung, Yong-Jun Choi

Department of Family Medicine, College of Medicine, the Catholic University of Korea, Seoul (JHL); Department of Family Medicine, Dongguk University IIsan Hospital, Goyang (NJS); Department of Social and Preventive Medicine and Health Services Research Center, College of Medicine, Hallym University, Chuncheon (YJC)

Symposium No. SY2-0502 Primary Care System Development in Taiwan

Chyi-Feng Jan, Wen-Jing Liu, Tai-Yuan Chiu

Department of Family Medicine, National Taiwan University Hospital, College of Medicine, National Taiwan University, Taipei (CFJ, WJL, TYC)

Symposium No. SY2-0503 Health care system and primary care in China Harry H.X. Wang, Yu Ting Li, Xiu Jing Hu, Jia Ji Wang

Sun Yat-Sen University, Guangzhou (HHXW, YTL, XJH); Guangdong-provincial Primary Healthcare Association (JJW) 
Symposium No. SY2-0504 Primary Care System and Research Activities in Japan

Takuya Aoki

Department of Healthcare Epidemiology, School of Public Health in the Graduate School of Medicine, Kyoto University, Kyoto (TA)

Main task:

To share the information on trends in each country at key characteristics of the primary care system such as primary care governance, economic conditions of primary care, primary care workforce development, and primary care process. Furthermore, we will discuss research themes that are currently being addressed or important in the future based on the situations of each country.

Implications:

Health systems based on primary care have been shown to achieve better health outcomes, equity, and costs. Therefore, many countries have been making efforts through primary care reforms to strengthen the care delivery. Especially in Europe and North America, efforts of exchange of experiences and health service researches through international collaboration have been active for the improvement of primary care quality. In East Asia, because such efforts have been poor, we have established the East Asian Primary Care Research Network (EAPCRN) at WONCA World 2018 Seoul. We expect this symposium can be a stepping stone to the successful development of the EAPCRN.

References:

https://confit.atlas.jp/guide/event/woncaaprjpca2019/session/SY2-05/category

\section{0 - Workshop titled "Current status of education and training in primary care in four different health systems across East Asia - a workshop from the East Asian Primary Care Research Network (EAPCRN)", Workshop No. 367 at the WONCA Asia Pacific Regional Conference (Auckland, New Zealand, 2020)}

\section{Presenters:}

Jae-Ho Lee, The Catholic University of Korea, Seoul

Chyi-Feng Jan, National Taiwan University, Taipei

Harry H.X. Wang, Sun Yat-Sen University, Guangzhou

Shin Yoshida, Lizuka Hospital, Fukuoka

\section{Main task:}

To present the contents on 1) Education and Training in Primary care in South Korea (Lee); 2) Family Medicine Education System in Taiwan (Jan), 3) General Practice Education and Training: a Primary Care Perspective from China (Wang); and 4) Recent reform in primary care education in Japan (Yoshida). Implications:

Primary healthcare (PHC) is an essential part of the healthcare system. PHC is usually the first point of contact people have with the health care system. It provides comprehensive, continuous, accessible, coordinated, and community-based care that meets the complex health needs of individuals throughout their life course. The general practitioner (GP)/ family physician (FP) plays one of the key roles in PHC. High-quality education and training is a prerequisite to teaching future GP/FPs. In this workshop, we will explain about current status of education and training for GP/FPs respectively in four different health systems across East Asia.

\section{Keywords}

East Asian; Primary Care; Research Network; EAPCRN

(C) 2020 by the author(s). Distribute under a Creative Commans CC BY license 